\title{
SIBBX20 interacts with the COP9 signalosome subunit SICSN5-2 to regulate anthocyanin biosynthesis by activating SIDFR expression in tomato
}

\author{
Dan Luo ${ }^{1}$, Cheng Xiong ${ }^{1}$, Aihua Lin ${ }^{1}$, Chunli Zhang ${ }^{1}$, Wenhui Sun ${ }^{1}$, Junhong Zhang ${ }^{1}$, Changxian Yang ${ }^{1}$, Yongen Lu', \\ Hanxia Li', Zhibiao Ye $\mathbb{I}^{1}$, Ping $\mathrm{He}^{2}$ and Taotao Wang ${ }^{1}$
}

\begin{abstract}
Anthocyanins play vital roles in plant stress tolerance and growth regulation. Previously, we reported that the photomorphogenesis-related transcription factor SIBBX20 regulates anthocyanin accumulation in tomato. However, the underlying mechanism remains unclear. Here, we showed that SIBBX20 promotes anthocyanin biosynthesis by binding the promoter of the anthocyanin biosynthesis gene SIDFR, suggesting that SIBBX20 directly activates anthocyanin biosynthesis genes. Furthermore, we found by yeast two-hybrid screening that SIBBX20 interacts with the COP9 signalosome subunit SICSN5-2, and the interaction was confirmed by bimolecular fluorescence complementation and coimmunoprecipitation assays. SICSN5 gene silencing led to anthocyanin hyperaccumulation in the transgenic tomato calli and shoots, and SICSN5-2 overexpression decreased anthocyanin accumulation, suggesting thSICSN5-2 enhanced the ubiquitination of SIBBX20 and promoted the degradation of SIBBX20 in vivo. Consistently, silencing the SICSN5-2 homolog in tobacco significantly increased the accumulation of the SIBBX20 protein. Since SIBBX20 is a vital regulator of photomorphogenesis, the SIBBX20-SICSN5-2 module may represent a novel regulatory pathway in light-induced anthocyanin biosynthesis.
\end{abstract}

\section{Introduction}

Anthocyanins are pigments synthesized by the flavonoid pathway involved in the coloring of various organs, such as leaves, fruits, and flowers ${ }^{1,2}$. Anthocyanins accumulate in response to plant hormones, low temperature, high temperature, strong light, UV-B radiation, and other environmental factors ${ }^{2-8}$. Moreover, fruit color is a significant indicator of quality. Anthocyanins are the main pigments responsible for determining color in a broad variety of fruits. Anthocyanins also play significant roles as antioxidants in plant biotic and abiotic stress

\footnotetext{
Correspondence: Taotao Wang (ttwang@mail.hzau.edu.cn)

${ }^{1}$ Key Laboratory of Horticulture Plant Biology, Ministry of Education, Huazhong Agriculture University, 430070 Wuhan, China

${ }^{2}$ Department of Biochemistry and Biophysics, Texas A\&M University, College Station, TX 77843, USA
}

tolerance and thereby facilitate plant resistance to pathogens and insects.

Aft and Atv are two important loci that regulate anthocyanin biosynthesis in tomato. The Aft gene from tomato was mapped to chromosome 10 and found to encode a SlAN2-like R2R3-MYB protein that promotes anthocyanin biosynthesis ${ }^{9,10}$. Atv is located on chromosome 7 and encodes the SIMYBATV protein, which negatively regulates the synthesis of anthocyanins. In addition to positively regulating anthocyanin biosynthesis, Aft was reported to directly activate SIMYBATV expression. Additionally, SIMYBATV competes with Aft for interaction with the transcription factor SIJAF13, thereby downregulating the accumulation of anthocyanins in tomato fruit. Mutation of SIMYBATV results in the release of SlJAF13, which interacts with Aft, further

\section{(c) The Author(s) 2021}

(c) (i) Open Access This article is licensed under a Creative Commons Attribution 4.0 International License, which permits use, sharing, adaptation, distribution and reproduction cc) in any medium or format, as long as you give appropriate credit to the original author(s) and the source, provide a link to the Creative Commons license, and indicate if changes were made. The images or other third party material in this article are included in the article's Creative Commons license, unless indicated otherwise in a credit line to the material. If material is not included in the article's Creative Commons license and your intended use is not permitted by statutory regulation or exceeds the permitted use, you will need to obtain permission directly from the copyright holder. To view a copy of this license, visit http://creativecommons.org/licenses/by/4.0/. 
leading to the upregulation of SlAN1 and SlAN11 expression and accumulation of anthocyanins in tomato fruit $^{9,11}$.

Anthocyanin accumulation is mostly regulated by transcription factors and structural genes, including $C H S$, CHI, F3H, F3' $H, F 3^{\prime} 5^{\prime} H, D F R, A N S$, and $U F G T^{8,12}$. The biosynthesis of anthocyanins is regulated by different transcription factors. The MYB-bHLH-WD40 (MBW) complex plays vital roles in regulating the biosynthesis of anthocyanins. The molecular mechanism by which the MBW complex regulates anthocyanin biosynthesis has been extensively studied. The WD40 protein likely plays a more general role in the regulation of the complex ${ }^{8,13}$. The activation of particular genes is determined by the expression pattern and DNA-binding specificity of the MYB and bHLH proteins.

SlAN2 was reported to promote anthocyanin biosynthesis when plants were grown in strong light and under low-temperature conditions ${ }^{14}$. A recent study showed that the overexpression of SIMYB75 induced the accumulation of anthocyanins ${ }^{15}$. Overexpression of SlANT1 was reported to increase the expression levels of structural genes in the anthocyanin biosynthesis pathway ${ }^{16}$. The MdMYB1/10 gene was found to be involved in regulating the accumulation of anthocyanins in apple ${ }^{17,18}$. The MYBTF gene Cs6g 17570 was identified as a vital player in the regulation of anthocyanin biosynthesis in blood oranges ${ }^{19}$. MdMYB16 and MdbHLH33 were also reported to be involved in anthocyanin metabolism ${ }^{20}$. In addition, MdbHLH3 was found to promote anthocyanin accumulation and fruit coloring under low-temperature conditions in apple ${ }^{21}$. The WD40 protein MdTTG1 was reported to interact with bHLH to regulate anthocyanin synthesis in apple ${ }^{22}$.

B-box (BBX) proteins are a class of zinc finger protein transcription factors that contain one or two B-box domains. Many studies in Arabidopsis have revealed that BBX family proteins play an important role in photomorphogenesis ${ }^{23}$. This particular group of BBX factors includes AtBBX1, AtBBX4, AtBBX19, AtBBX20, AtBBX21, AtBBX22, AtBBX23, AtBBX24, AtBBX25, AtBBX28, AtBBX30, AtBBX31 and AtBBX32 $2^{24-31}$. The accumulation of anthocyanin is a general phenomenon in photomorphogenesis, and BBX proteins were also found to regulate anthocyanin synthesis. In pear, the BBX proteins PpBBX16, PpBBX18, PpBBX21, and PpBBX24 are involved in anthocyanin accumulation $^{5,6,32}$. In apple, MdCOL4, MdBBX20, MdBBX22, and MdBBX37 were found to participate in the regulation of anthocyanin accumulation ${ }^{2-4,33}$.

In a previous study, we found that the SlBBX20 protein is modified by the CRL4 E3 ubiquitin ligase to regulate the biosynthesis of carotenoids in tomato fruit ${ }^{34}$. In addition to the carotenoid content, we found that overexpression of the $S l B B X 20$ gene led to a significant increase in the anthocyanin content. Here, SlBBX20 was found to target the $D F R$ promoter and activate its expression. To further uncover the mechanism by which anthocyanin biosynthesis is regulated, we screened a yeast two-hybrid library and found that SlCSN5-2 interacts with SlBBX20. The downregulation of SICSN5-2 resulted in the accumulation of anthocyanin in tomato. Furthermore, when we interfered with the expression of a SlCSN5-2 homolog in tobacco, the expression level of the SIBBX20 protein was significantly increased, indicating that SICSN5-2 regulates the accumulation of the SlBBX20 protein.

\section{Results}

\section{Overexpression of SIBBX20 led to increased anthocyanin accumulation}

To study the role of SlBBX20 in regulating the accumulation of anthocyanins, we overexpressed full-length SlBBX20 in tomato with the $35 \mathrm{~S}$ promoter. In the transformation process, we found that the partial calli growing on the medium had become purple (Fig. 1a). After rooting, the seedlings and roots of the SlBBX20overexpressing plants were also purple (Fig. 1b, c). Purple sepals in the flowers of SlBBX20-overexpressing plants were also observed (Fig. 1d). Thus, anthocyanins accumulated in diverse tissues with the overexpression of SlBBX20.

We also obtained three homozygous SIBBX20-knockout plants using CRISPR-Cas9 technology. No obvious difference in anthocyanin content was observed between the knockout plants and control plants, which may be due to redundant gene functions. Recent studies have shown that Arabidopsis $B B X 20,21$ and 22 are functionally redundant and regulate hypocotyl elongation and anthocyanin accumulation as rate-limiting cofactors of $\mathrm{HY}^{35}$. Therefore, we selected SlBBX20-overexpressing plants to perform follow-up experiments.

We quantified the expression of SlBBX20 and measured the anthocyanin content in 14 independent transgenic lines (Fig. 2a-c). The level of SlBBX2O transcription was highly correlated with anthocyanin content. We further used the Pearson correlation coefficient to assess the correlation between these two parameters (Fig. 2d). The scatter plot showed the Pearson correlation coefficient between the relative SIBBX2O expression level and level of accumulated anthocyanin to be 0.84 , indicating a strong positive correlation between them.

\section{The expression of flavonoid biosynthesis genes was upregulated in SIBBX20-overexpressing lines}

To explore the molecular mechanism by which SIBBX20 regulates anthocyanin accumulation, we analyzed the transcriptomes of the $S I B B X 20$-overexpressing plants and controls by RNA sequencing (RNA-Seq). 

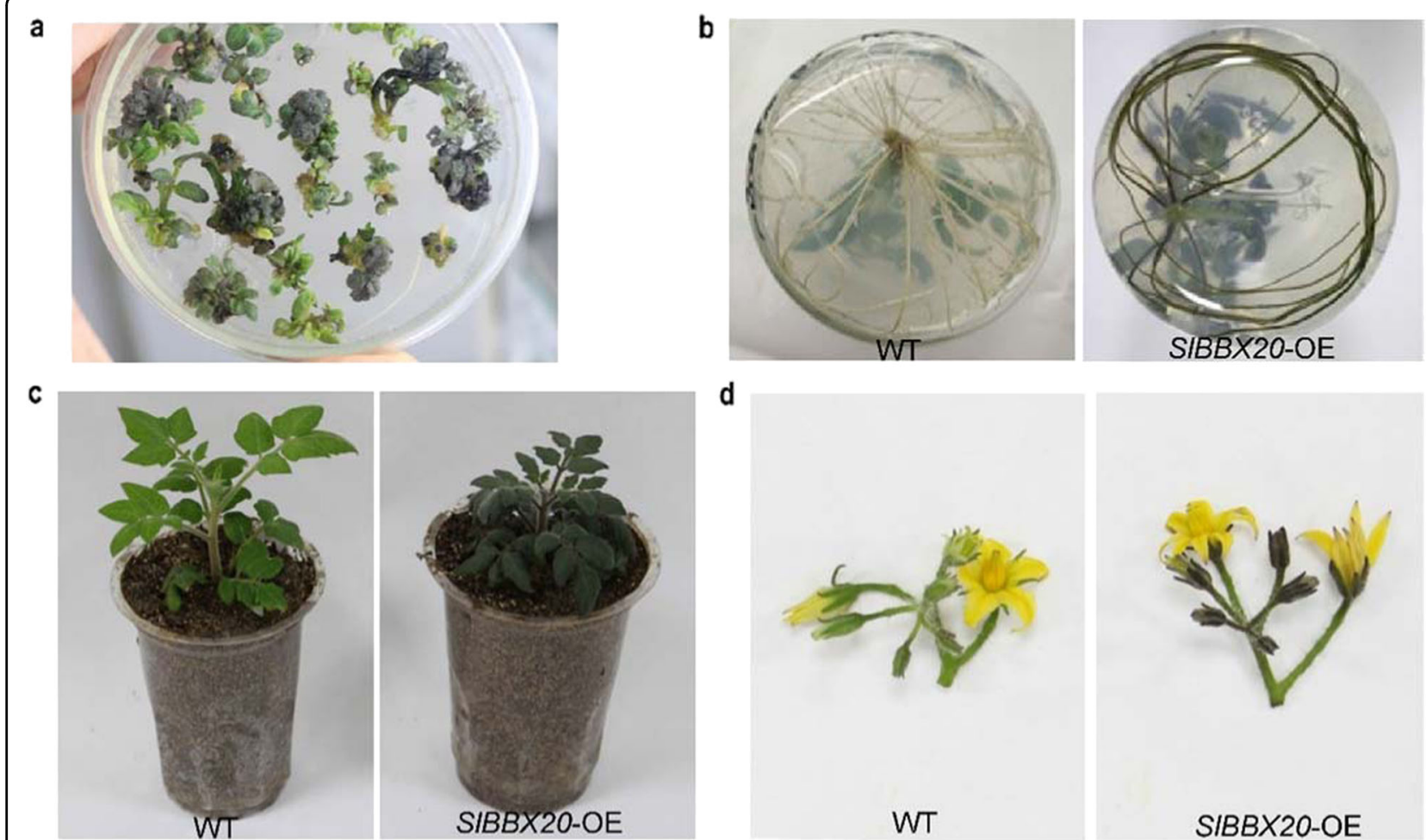

d

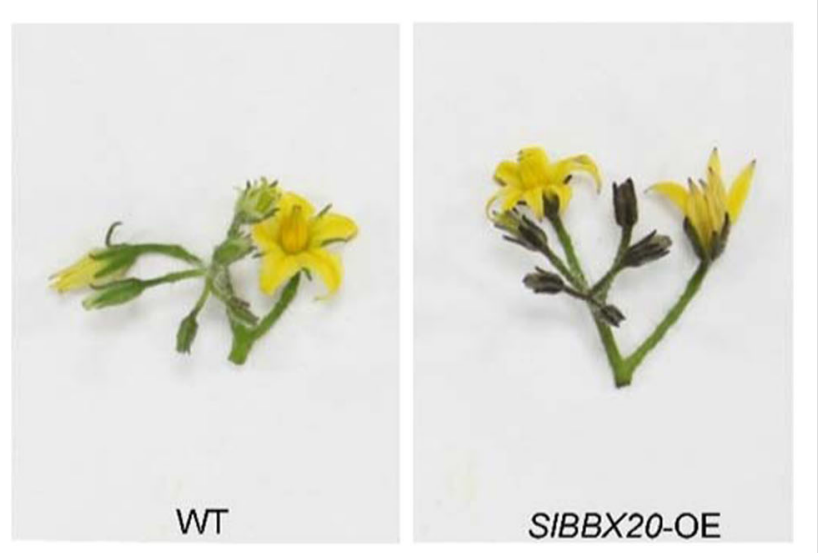

Fig. 1 Anthocyanin accumulation in SIBBX20-overexpressing tomato lines. SIBBX20 overexpression enhanced anthocyanin biosynthesis in the calli and shoots (a), roots (b), seedlings (c), and flowers (d) compared with that in the wild-type (WT) plants

Anthocyanins are synthesized by the flavonoid pathway. The expression levels of some genes in the pigment, flavonoid and anthocyanin biosynthesis pathways (DFR, $A N S$, CHS1, CHS2, F3H, F3'5' H, and $F L S$ ) were found to be upregulated in the $S I B B X 20$-overexpressing plants (Fig. 3a). To independently validate these results, we quantified the expression of these anthocyanin-related structural genes in two SlBBX20-overexpressing lines, OE-20 and OE-40 (Fig. 3b). The expression levels of CHS1, CHS2, F3H, F3'5'H, DFR, and FLS were found to be upregulated in the SIBBX20-overexpressing lines relative to the control line. Among these genes, DFR, ANS, and $F 3^{\prime} 5^{\prime} H$ were elevated more than twofold. This result is consistent with the transcriptome data, and these data imply that $S I B B X 20$ promotes the accumulation of anthocyanins by regulating the expression of anthocyanin biosynthesis genes.

\section{SIBBX20 directly regulates SIDFR}

We hypothesized that SIBBX20 directly regulates transcription of the anthocyanin biosynthesis genes that were upregulated in the SlBBX20-overexpressing lines. BBX proteins were reported to regulate their target genes by binding G-boxes in their promoters. The cis-elements in the promoters of DFR, ANS, CHS1, CHS2, F3H, F3'5' H, and $F L S$ were analyzed by using the PlantCARE database (http://bioinformatics.psb.ugent.be/webtools/plantcare/ $\mathrm{html} /)^{36}$. The promoter of ANS does not contain a G-box. Therefore, we speculated that SIBBX20 cannot bind the ANS promoter. Yeast one-hybrid assays were conducted to test whether SIBBX20 regulates the other genes in this group. The yeast strain Y1GOLD was cotransformed with $A D-S l B B X 20$ and $p A b A i-C H S 1, p A b A i-C H S 2, p A b A i-$ $F 3 H, p A b A i-F 3^{\prime} 5^{\prime} H, p A b A i-F L S$ or a negative control. None of these transformants survived on the selective medium, which lacked Leu and Ura and contained AbA. These data indicate that SIBBX20 could not interact with the promoters of CHS1, CHS2, F3H, F3'5'H, or FLS, although these promoters contain G-boxes (data not shown). The promoter of SIDFR contains three G-boxes (Fig. 4a). We designed the G-box1, 2 and 3 sequences to confirm their interaction. We found that Y1GOLD yeast cells cotransformed with $A D-S l B B X 20$ and $p A b A i-S I D F R$ (including G-box1, 2) could survive on the selective medium. However, $p A b A i-S l D F R$ including G-box 3 could not survive on the medium (data not shown). These data indicate that SlBBX20 can bind G-box1 or 2 in the promoter of SIDFR (Fig. 4b).

Next, we performed an EMSA to determine which Gbox (G-box1 or 2) is targeted by SlBBX20. In the EMSAs, the SlBBX20 protein bound the G-box1-wt probe but not the other probes. This result demonstrated that SlBBX20 


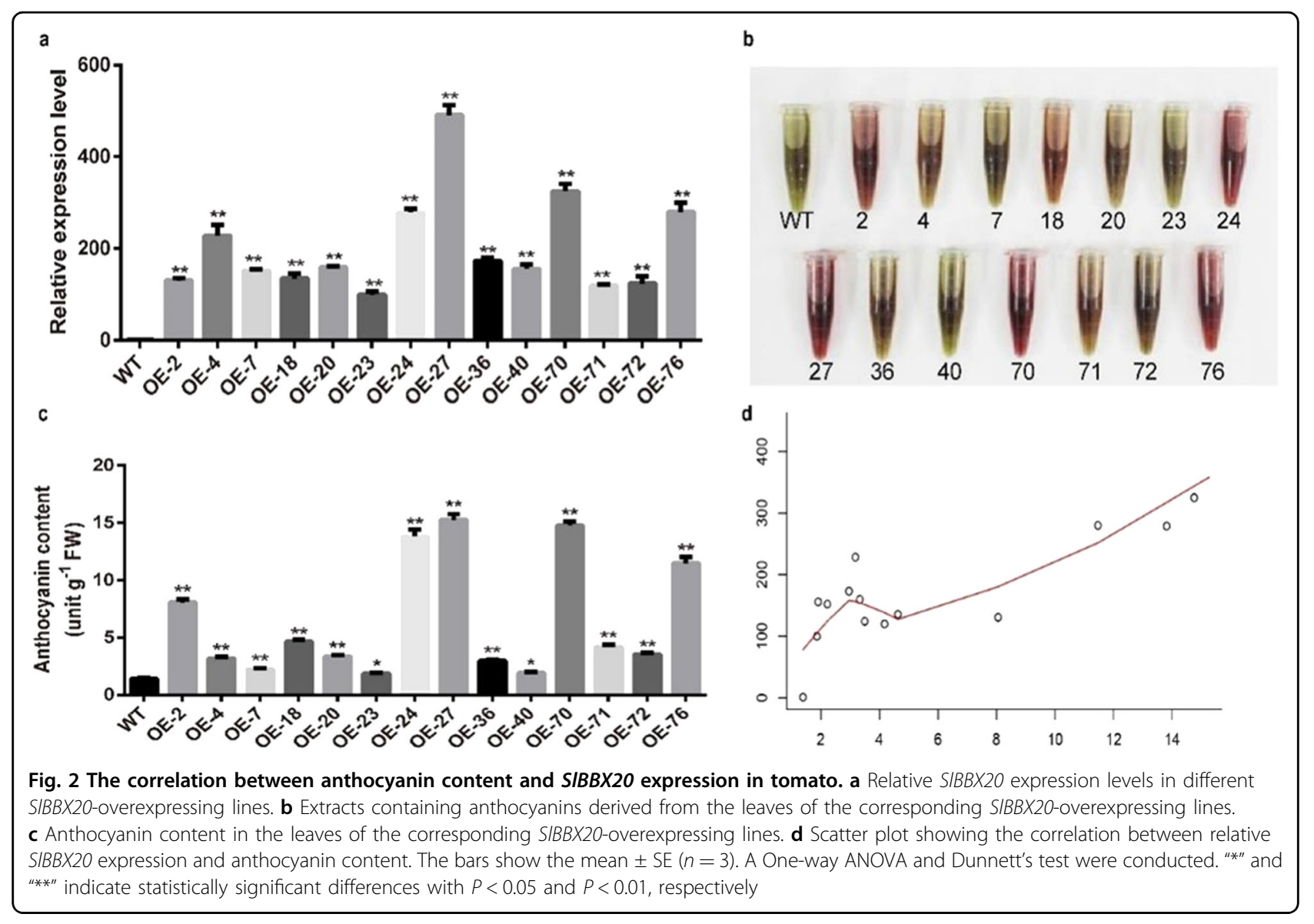

binds G-box1 in the SIDFR promoter in vitro (Fig. 4c, d). Subsequently, a dual-luciferase system assay was performed to test whether SIBBX20 could activate the expression of SIDFR. As shown in Fig. 4f, the ratio of LUC to REN in tobacco leaves co-injected with $62 S K-S I B B X 20$ and $p A b A i-S I D F R$ was increased by 2.6 -fold relative to the negative control (62SK and $p A b A i-S I D F R)$. These results provide evidence that SIBBX20 can activate the transcription of SIDFR by binding the G-box1 cis-element in its promoter.

\section{SIBBX20 interacts with SICSN5-2 in vivo}

To further analyze the molecular mechanism by which SIBBX20 regulates anthocyanin content, a yeast twohybrid $(\mathrm{Y} 2 \mathrm{H})$ screen was performed using SlBBX20 as bait. We found that the protein encoded by Solyc06g073150 could interact with SlBBX20 in the Y2H screen. The full-length coding sequence of this gene is $1104 \mathrm{bp}$ in length. The gene encodes a predicted protein consisting of 367 amino acid (aa) residues, which was referred to as SICSN5-2 in a previous study ${ }^{37}$. We used three different methods to confirm the interaction between the SlBBX20 and SICSN5-2 proteins. First, the interaction was verified using the $\mathrm{Y} 2 \mathrm{H}$ assay (Fig. 5a).
The full-length SlCSN5-2 protein was previously demonstrated to generate false-positive results in the $\mathrm{Y} 2 \mathrm{H}$ assay. Therefore, a truncated SlCSN5-2 construct (SlCSN5-2 27-367) was used to test the interaction. SlBBX20 was also divided into two fragments consisting of residues 56-203 or 101-203. The yeast cells cotransformed with the $B D-S l B B X 20 / B D-S l B B X 20_{56-203}$ and AD-SlCSN5-257-367 plasmids grew on SD -Leu/Trp/His/Ade medium, but the negative control yeast cells and yeast cells cotransformed with $B D-S l B B X 20_{101-203}$ and AD-SlCSN5-2 $57-367$ did not grow on this medium, suggesting an interaction

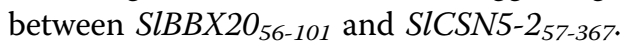

Second, we employed a coimmunoprecipitation assay to confirm the interaction (Fig. 5b). SlBBX2O-HA and SICSN5-2-FLAG plasmids were cotransformed into tobacco protoplasts and expressed for $8-10 \mathrm{~h}$. The proteins were immunoprecipitated using an anti-FLAG antibody and immunoblotted using an anti-HA antibody. The SIBBX20-HA protein was coimmunoprecipitated with SICSN5-2-FLAG, but the negative control was not (Fig. 5b).

Finally, the interaction between SlBBX20 and SlCSN5-2 was tested using the bimolecular fluorescence complementation (BiFC) assay. We observed YFP fluorescence 

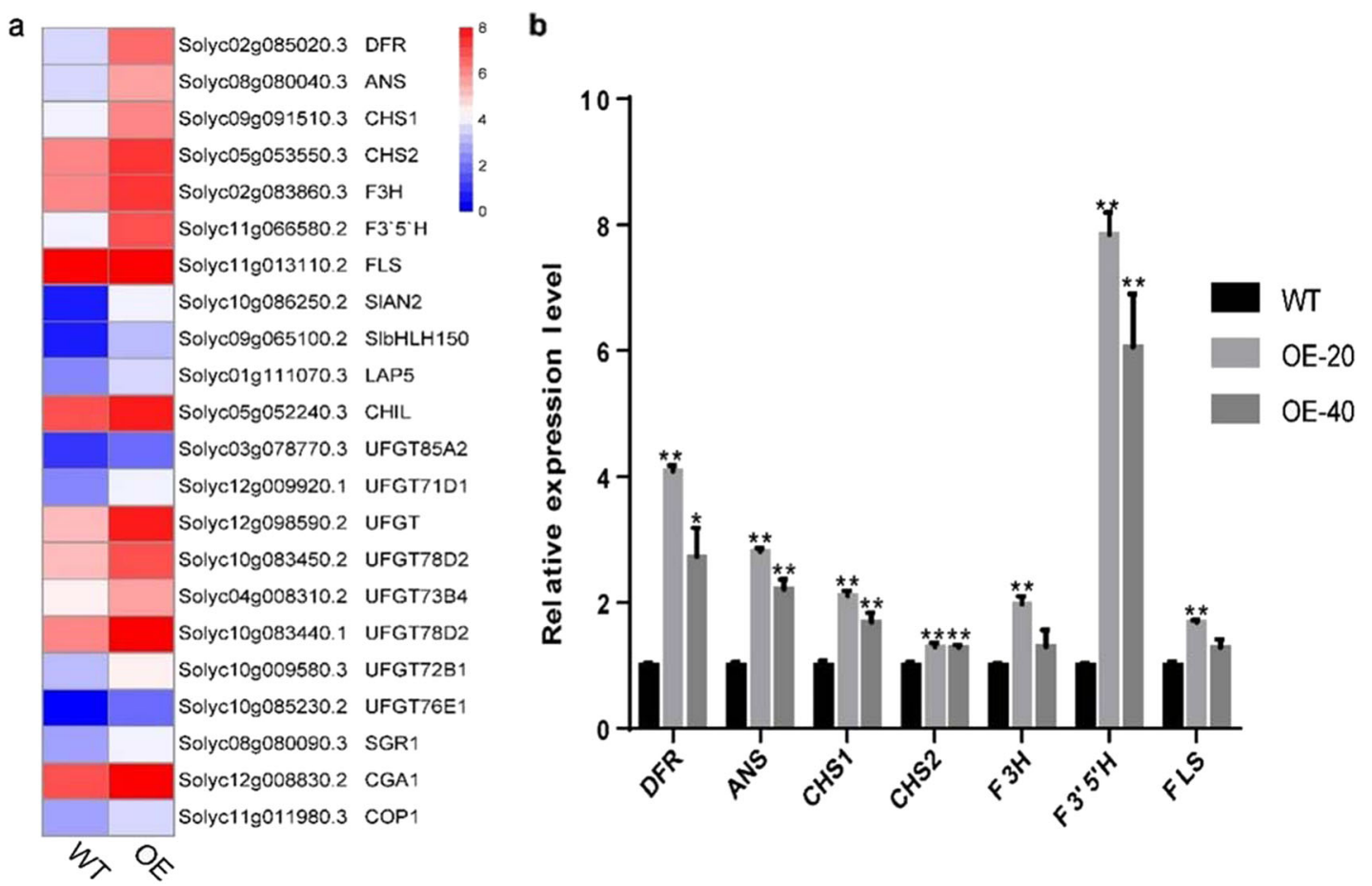

Fig. 3 Expression of genes associated with pigment biosynthesis obtained from transcriptome data. a Heatmap of pigment-associated genes with increased expression in SIBBX20-overexpressing lines identified from an analysis of transcriptome data. $\mathbf{b}$ Validation of the transcriptome data using qRT-PCR. We validated the expression levels of structural genes associated with anthocyanin biosynthesis that were classified as upregulated based on an analysis of our transcriptome data

in tobacco protoplast cells cotransformed with the SlBBX20-nYFP and SlCSN5-2-cYFP plasmids but not in the negative control tobacco protoplast cells cotransformed with the empty vector and SlBBX20-nYFP or SlCSN5-2-cYFP plasmids (Fig. 5c). These data provide in vivo evidence that SlBBX20 interacts with SICSN5-2.

We also determined the subcellular localization of SICSN5-2, and the results showed that SICSN5-2 accumulates in both the nucleus and cytoplasm (Fig. 5d). This localization pattern was very similar to that of SlBBX20 34 , suggesting that SICSN5-2 and SlBBX20 work together.

\section{SICSN5 regulates anthocyanin accumulation}

To study the effects of SlCSN5-2 on anthocyanin biosynthesis, we used RNA interference to downregulate the expression of SlCSN5 (including SlCSN5-1 and SlCSN5-2) in stably transformed plants. It was impossible to independently silence SlCSN5-1 and SlCSN5-2 in tomato due to their high sequence similarity ${ }^{37}$. While generating the transgenic plants, we observed many purple calli and shoots (Fig. 6a), similar to the effects of SlBBX2O overexpression. Strongly SlCSN5-silenced calli accumulated many anthocyanins but failed to grow into normal plants. The moderately silenced plants survived, but their growth was significantly restrained (Fig. 6b, c), indicating that the function of SlCSN5-2 in growth and development is indispensable. We selected a line in which SlCSN5 expression was moderately decreased to test the anthocyanin content and found that it was significantly increased compared with that in the WT plants (Fig. 6d). Correspondingly, when SlCSN5-2 was overexpressed in tomato, the anthocyanin content was significantly decreased compared with that in the WT plants (Fig. 6e), suggesting that SlCSN5 is a negative regulator of anthocyanin biosynthesis.

Furthermore, to confirm whether SlCSN5-2 regulates the accumulation of anthocyanin by $D F R$, we detected the expression level of SIDFR in SlCSN5-RNAi plants. The expression level of SIDFR was significantly upregulated compared with that of the WT plants, and the expression level of SlCSN5-2 was negatively correlated with that of SIDFR (Fig. 6f). This result suggests that SlCSN5 negatively regulates the accumulation of anthocyanin in tomato by SIDFR.

\section{SICSN5-2 regulates accumulation of the SIBBX20 protein}

Previously, we revealed that SIBBX20 is modulated by the E3 ubiquitin ligase CRL4 $4^{34}$. SICSN5-2 was reported to regulate CRL ubiquitin ligase and may regulate 


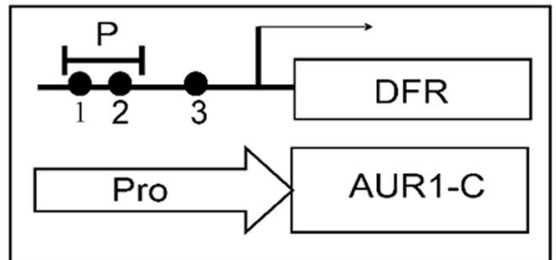

b

$-A b A$

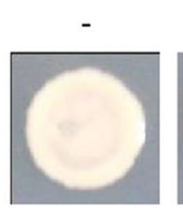

$+\mathrm{AbA}$
SIBBX20
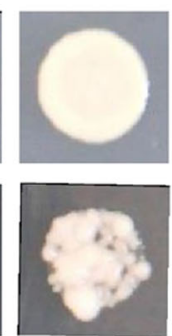

c Gbox1-wt: TATGATTAGTGAAAGACCAACGTGTTTGAATGGATCCAGTTC Gbox1-mt: TATGATTAGTGAAAGACCGGGGGGTTTGAATGGATCCAGTTC Gbox2-wt: AGGAGTCTTAATTAACAGCACATGGTTAATTTTTCTTCTGAAA Gbox2-mt: AGGAGTCTTAATTAACAGGGGGGGGTTAATTTTTCTTCTGAAA

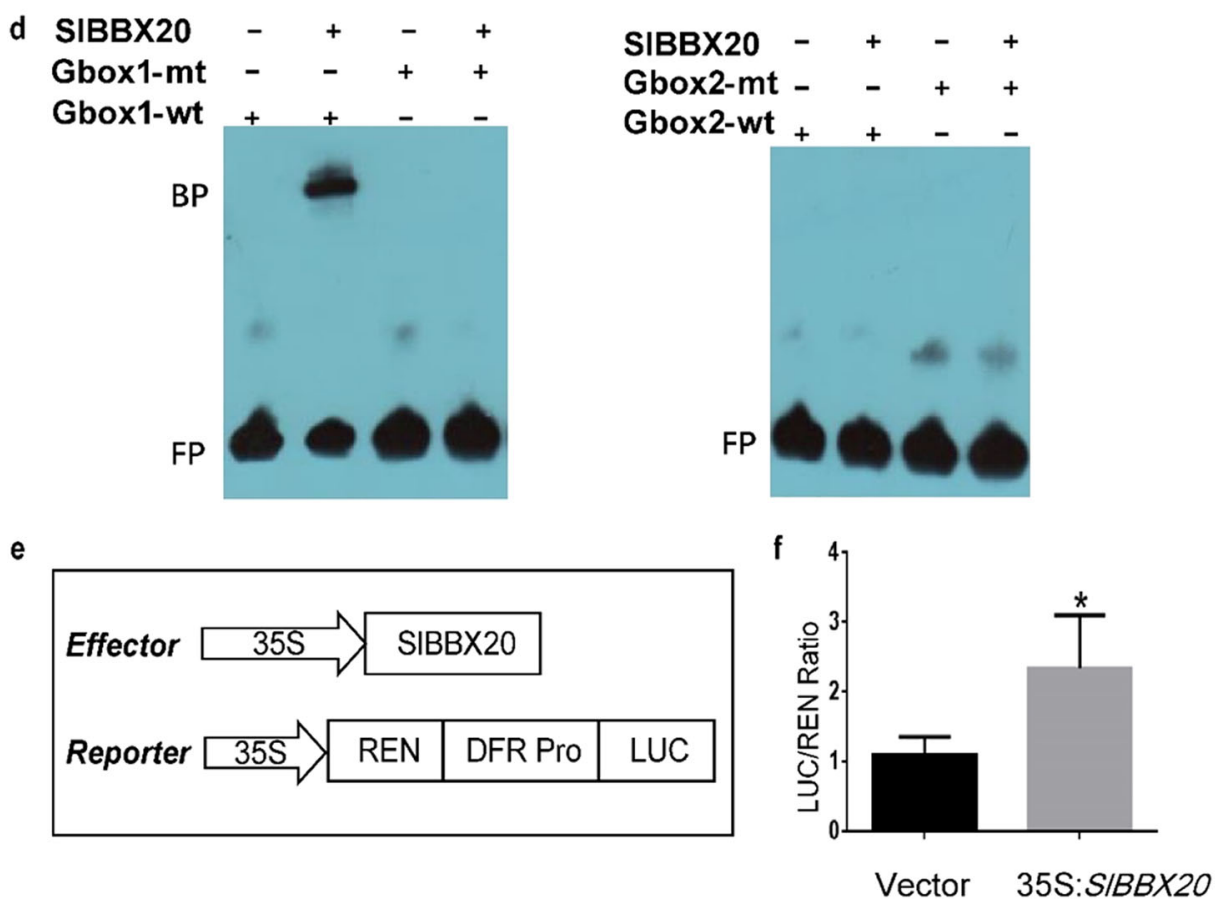

Fig. 4 SIBBX20 activates the expression of DFR by binding its promoter. a Schematic diagram of the G-box locations on the DFR promoter. P1 includes G-box1 and G-box2, and the black dots indicate the locations of the G-boxes. b SIBBX20 was shown to bind the DFR promoter in a yeast one-hybrid assay. The yeast strain Y1HGold was transformed with the bait vector PAbAi-DFR and the prey vector AD-SIBBX20 and plated on SD -LeuUra medium with or without aureobasidin A $\left(45 \mathrm{ng} \mathrm{mL}^{-1}\right)$. c Wild-type and mutant probe sequences used for EMSAs. G-box 1-wt and G-box2-wt were used as wild-type probes. The synthesized G-box1-mt and G-box2-mt sequences were mutation probes in which the cis-element sequences were replaced by GGGGGG or GGGGGGG, respectively. $\mathbf{d}$ Affinity of SIBBX20 for two G-box motifs. BP indicates the binding probe; FP indicates the free probe. " + " and "-" indicate presence or absence in the EMSA, respectively. e Schematic of the vector used for the dual-luciferase experiment. f LUC/REN ratios. Luciferase activity was detected by dual-luciferase reporter assay

ubiquitination of the SIBBX20 protein by modifying the E3 ubiquitin ligase CRL4. To explore whether SICSN5-2 affects ubiquitination of the SlBBX20 protein, we conducted an immunoprecipitation assay to detect the ubiquitination of SlBBX20 with an anti-UBQ antibody. The results showed that upon SlCSN5-2 coexpression, ubiquitination of the SIBBX20 protein was enhanced, and expression of the SIBBX20 protein decreased with actin used as an input control (Fig. 7a).
$N b C S N 5 B$ in tobacco is an ortholog of SlCSN5-2, and their gene sequences are highly similar (Supplementary Fig. S1). We used VIGS to silence NbCSN5B in tobacco. Relative $N b C S N 5 B$ gene expression was confirmed to be downregulated in leaves (Fig. 7b). Then, we extracted protoplasts from tobacco and transiently expressed SlBBX20-HA in the protoplasts. After $10 \mathrm{~h}$, the proteins were extracted and detected by western blotting (Fig. 7c). We found that the SIBBX20-HA protein accumulated to 


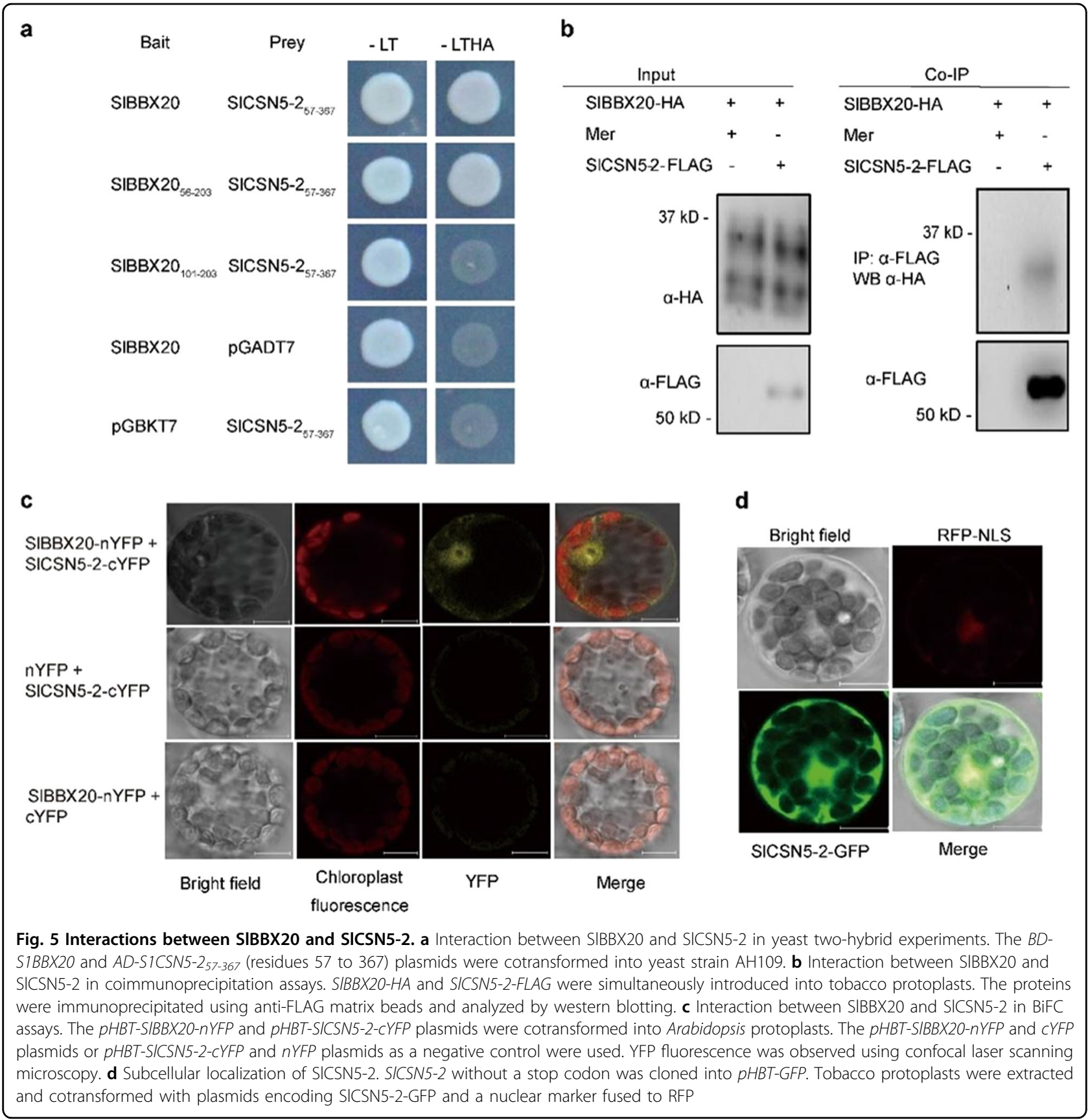

higher levels in the $N b C S N 5 B$-silenced plants than in the control plants. In general, these results suggest that SICSN5-2 interacts with SIBBX20 and promotes its ubiquitination and degradation to negatively regulate anthocyanin biosynthesis.

\section{Discussion}

Anthocyanins are natural plant pigments involved in regulating the coloration of specific plant organs, such as leaves, flowers, and fruits. Previous studies on the regulation of anthocyanin biosynthesis have mainly focused on the MBW complex. Some positive regulators of anthocyanin biosynthesis, such as SlAN2, SlAN1, and SIMYB7 $5^{14,15}$, and some negative regulators of anthocyanin biosynthesis, such as MdMYB16, FaMYB1, and PhMYB27 ${ }^{20,38,39}$, have been reported in different plants. Recently, some BBX proteins in apple (i.e., MdCOL4, MdBBX20, MdBBX22) were found to regulate anthocyanin biosynthesis ${ }^{2-4,33}$. In pear, PpBBX16, PpBBX18, $\mathrm{PpBBX} 21$, and PpBBX24 were reported to be involved in anthocyanin synthesis ${ }^{5,6,32}$. A recent study showed that MdBBX37 inhibits the transactivating activities of 

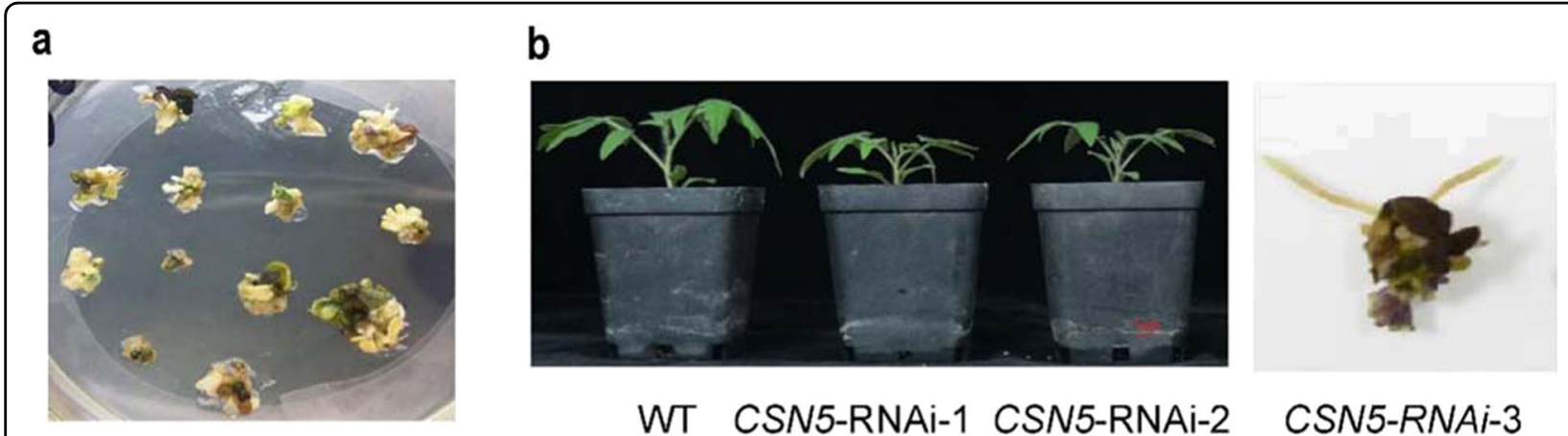

WT CSN5-RNAi-1 CSN5-RNAi-2

CSN5-RNAi-3
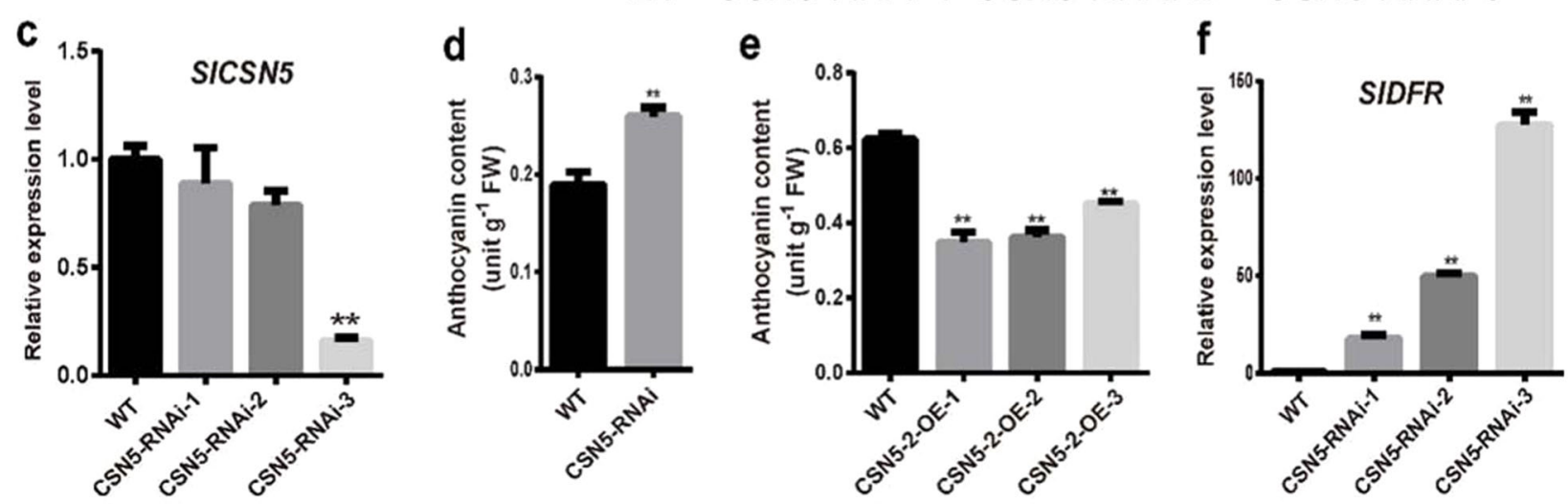

Fig. 6 SICSN5 regulates anthocyanin accumulation. Calli (a) and seedlings (b) after SICSN5 interference. c Detection of SICSN5 expression in the SICSN5-RNAi seedlings. d Anthocyanin content in the SICSN5-RNAi plants. e Anthocyanin content in the SICSN5-2-overexpressing plants. f The relative expression level of SIDFR in the SICSN5-RNAi seedlings. Data represent the mean \pm SE $(n=3)$. One-way ANOVA was performed. "** and "**" indicate statistically significant differences with $P<0.05$ and $P<0.01$, respectively

MdMYB1 and MdMYB9 and therefore downregulates anthocyanin biosynthesis ${ }^{3}$.

In this study, we found that SIBBX20 interacts with SICSN5-2 and promotes anthocyanin biosynthesis by binding the $D F R$ promoter. Tomato plants overexpressing SIBBX20 accumulated high levels of anthocyanins. Anthocyanins are synthesized by the flavonoid pathway, which contains genes that contribute to anthocyanin biosynthesis at both the early and late stages ${ }^{40}$. We found that most of these genes were upregulated in the SlBBX20-overexpressing lines, although the extent to which they were upregulated varied. BBX transcription factors were reported to regulate transcription by binding G-box cis-elements ${ }^{4,6,33}$. Here, SIBBX20 was found to directly bind the first G-box in the SIDFR promoter and activate its expression, and the accumulation of anthocyanins was highly correlated with the expression level of SIBBX20. DFR is a key enzyme in the anthocyanin synthesis pathway, its mutation blocks the accumulation of anthocyanin in tobacco, and a white flower phenotype appeared $^{41}$. MdBBX20 was reported to promote anthocyanin biosynthesis by binding the promoters of $D F R$, $A N S$, and $M Y B 1^{2}$. PpBBX16 requires PpHY5 to increase the expression levels of genes related to anthocyanin biosynthesis ${ }^{5}$. Thus, the mechanisms used by BBX20 to promote anthocyanin biosynthesis appear to be similar but vary among different plant species.

A large number of studies in Arabidopsis have shown that BBX family proteins are involved in photomorphogenesis ${ }^{26-31}$. Our previous study showed that SIBBX20-overexpressing tomato plants exhibited enhanced photomorphogenesis ${ }^{34}$. We identified the SICSN5-2 protein as a binding partner of SIBBX20. The COP9 signalosome (CSN) plays an important role in plant photomorphogenesis and was originally discovered by cloning mutant alleles that disrupt photomorphogenesis in Arabidopsis ${ }^{42}$. The accumulation of anthocyanins is an important phenomenon in photomorphogenesis. Recently, several BBX proteins were found to interact with the HY5 protein and regulate anthocyanin accumulation in Arabidopsis, apple, and pear ${ }^{3,6,35}$. UV-B radiation induces the accumulation of anthocyanins using a signaling mechanism that depends on MdCOP1; This mechanism activates MdHY5 and promotes the binding of MdHY5 to the MdMYB gene-promoter region ${ }^{7}$. In this study, we found that CSN5-2-a photomorphogenesis factor-could negatively regulate the accumulation of anthocyanins by promoting the accumulation of the SIBBX20 protein. Although the SlBBX20-OE plants accumulated many anthocyanins under light, anthocyanin 
a

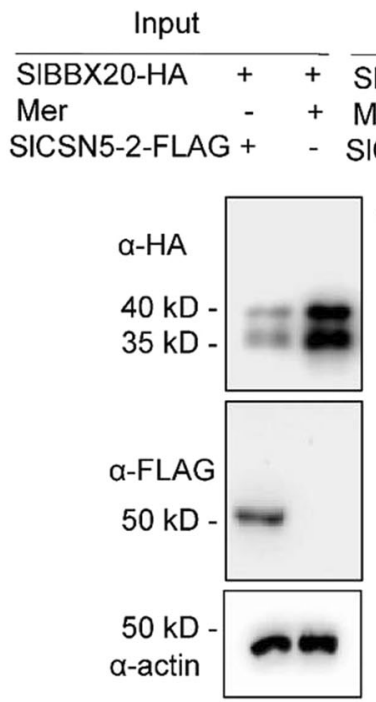

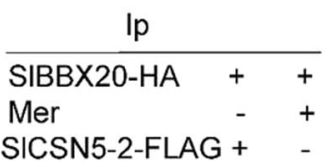

IP $\alpha-F L A G$

WB $\alpha-\mathrm{HA}$

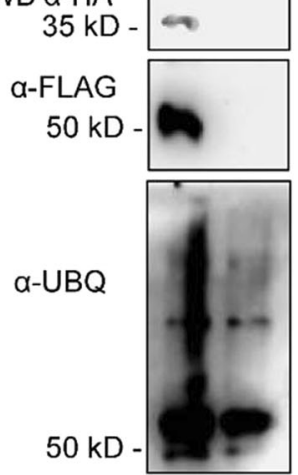

b

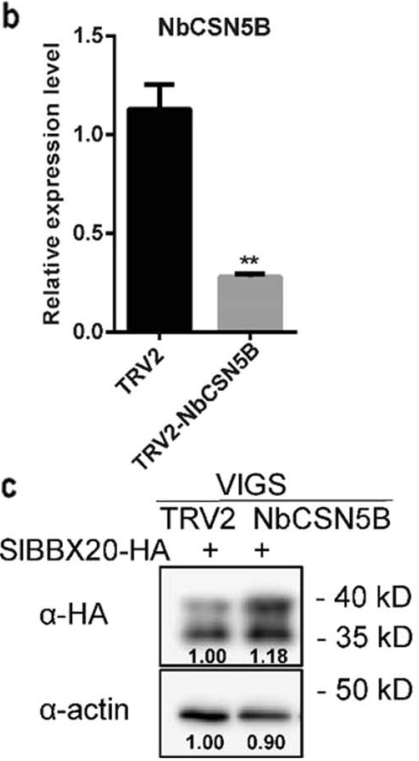

Fig. 7 SICSN5-2 regulates accumulation of the SIBBX20 protein. a The effect of SICSN5-2 on ubiquitination of the SIBBX20 protein. The SIBBX20HA and SICSN5-2-FLAG plasmids were cotransformed into tobacco protoplasts. Anti-FLAG matrix beads were used for immunoprecipitation experiments. Finally, several antibodies (anti-HA, anti-FLAG, and anti-UBQ) were used to detect the accumulation of different proteins, and actin was used as a control. b Relative NbCSN5B expression level in NbCSN5B-VIGS plants. Tobacco leaves were infiltrated with pTRV2-NbCSN5B and pTRV2 as controls. C NbCSN5B downregulated the SIBBX20-HA protein. NbCSN5B was silenced in tobacco by virus-induced gene silencing. The protoplasts were extracted and assessed using anti-HA, TRV2 as a control

accumulation in the SIBBX20-OE plants was reduced upon exposure to dark conditions for a period of time, and the expression of SIDFR was significantly downregulated compared with that under light (Supplementary Fig. S2a, b, d). suggested that the anthocyanin modulation by SIBBX2O is dependent on light. The transcription level of SlCSN5-2 was not significantly changed when the plants were exposed to dark conditions (Supplementary Fig. S2c). Previous studies have indicated that the protein level of COP9 is not affected by light, but light may regulate the activity of the SlCSN5-2 protein at the posttranslational level $^{43}$. Therefore, we believe that light is required for anthocyanin regulation in the SlBBX20-SICSN5-2 model. These data reveal a novel regulatory pathway involved in light-induced anthocyanin biosynthesis.

Interestingly, when we knocked down the expression of SlCSN5 in tomato using RNAi, anthocyanins accumulated in the calli and shoots, and strongly silenced plants accumulated abundant anthocyanins but failed to grow into normal plants. A moderate decrease in the level of SlCSN5 expression led to dwarfing, indicating the important function of SlCSN5 in tomato development. Tomato contains two CSN5 genes, namely, SlCSN5-1 and SlCSN5-2. Because their sequences are highly similar, it is difficult to individually interfere with the expression of these genes. Indeed, previous studies also reported that it was impossible to use different sequences to separately silence the two CSN5 genes in tomato ${ }^{37}$.
SlCSN5-VIGS plants were reported to be approximately $50 \%$ shorter in stature than controls ${ }^{37}$. The effects of SlCSN5 on growth and development may have been more severe in our stably transformed tomato lines. Among Arabidopsis thaliana, the csn 5 a mutant develops purple cotyledons ${ }^{44}$, but the reason is unknown. We found the sequence similarity between SlCSN5-2 and AtCSN5a to be $84 \%$. We speculate that AtCSN5a utilizes a mechanism to regulate anthocyanin biosynthesis that is similar to that of SICSN5-2.

CSN can regulate protein degradation through the ubiquitination pathway. The major activity of CSN is regulated by the fifth subunit $(C S N 5)^{45}$. CSN5 has been reported to be involved in deneddylation activity ${ }^{46}$. It can regulate the activity of CRLs (Cullin RING ligases) by covalently binding and removing RUB proteins ${ }^{47,48}$. Ubiquitinated proteins have been reported to accumulate in Arabidopsis csn mutants ${ }^{49}$. In the present study, we found that the ubiquitination of SIBBX20 was enhanced when it was coexpressed with SlCSN5. Furthermore, when we silenced the SlCSN5 homolog NbCSN5B in tobacco, accumulation of the SlBBX20 protein increased, indicating that CSN5 regulates accumulation of the SlBBX20 protein. Therefore, we infer that CSN5 is involved in ubiquitination and degradation of the SIBBX20 protein. Our previous study demonstrated that SIBBX20 can be ubiquitinated by the CUL4-DET1-DDB1 complex and eventually degraded by the $26 \mathrm{~S}$ proteasome ${ }^{34}$. CSN was 
found to modify the activity of several CUL4-based E3 ubiquitin ligases to regulate plant photomorphogenesis ${ }^{50}$. CSN5 might participate in regulating the activity of CUL4-based E3 ubiquitin ligases ${ }^{51}$. However, how CSN5 regulates the activity of CRLs and the accumulation of substrate remains unknown. Our work might provide insight into the modification of CSN5 to CRLs.

\section{Materials and methods \\ Plant materials}

The SIBBX20 gene was cloned into the overexpression vectors pHellsgates and $p C A M B I A 2300-H A$. Using the "Alisa Craig" (LA2838A) tomato as the wild-type background, transgenic tomato plants were obtained by Agrobacterium tumefaciens-mediated transformation. The expression of SlBBX2O in the transgenic plants was quantified using qRT-PCR.

\section{Gene expression analysis}

TRIzol reagent (Invitrogen, USA) was used to extract total RNA from leaves as previously described ${ }^{52}$. cDNA was synthesized using a HiScript ${ }^{\circledR}$ II 1 st Strand cDNA Synthesis Kit (Vazyme, China). Gene-specific oligonucleotides were used to perform qRT-PCR in a Roche LightCycler 480 system $^{53}$. Relative gene expression was calculated by Microsoft Excel. Expression of the actin gene (SGN-U580609) was used as an internal control. The sequences of the gene-specific oligonucleotides used in the analysis are listed in Supplementary Table S2.

\section{Measurement of the total anthocyanin content}

The methanol- $\mathrm{HCl}$ method was used to extract anthocyanins from tomato leaves. Approximately $2 \mathrm{~g}$ of tomato leaves ground with liquid nitrogen was soaked in $5 \mathrm{ml}$ of $1 \%(\mathrm{v} / \mathrm{v})$ methanol $\mathrm{HCl}$ and extracted overnight in the dark at $24^{\circ} \mathrm{C}$. A spectrophotometer (UV-1600, Shimadzu, Japan) was used to measure the absorbance of each sample at 530, 620, and $650 \mathrm{~nm}$. The following formula was used to calculate the relative anthocyanin content: optical density $(\mathrm{OD})=\left(\mathrm{OD}_{530}-\mathrm{OD}_{620}\right)-0.1$ $\left(\mathrm{OD}_{650}-\mathrm{OD}_{620}\right)$. One unit of anthocyanin represented a change in the OD of 0.1 .

\section{RNA sequencing}

Three biological replicates from 4-week-old tomato seedlings that overexpressed SIBBX2O and WT tomato seedlings were selected for the extraction of total RNA and RNA sequencing. We used the average RPKM (reads per kilobase per million reads) value as a measure of gene expression ${ }^{54}$. Genes showing at least a twofold change in expression with an FDR-adjusted p-value of less than 0.05 were defined as differentially expressed genes (DEGs). The heat map was plotted with $\log _{2}$ RPKM values to visually show differences in expression levels.

\section{Yeast one-hybrid assay}

The full-length $S I B B X 20$ gene was amplified with tomato cDNA as the template and inserted into $p G A D T 7$ to obtain the prey vector $(A D-S l B B X 20)$. Fragments of the DFR, CHS1, CHS2, F3H, F3'5'H, and FLS promoters were amplified with tomato genomic DNA as the template and cloned into $p A b A i$ to obtain a bait vector ( $p A b A i-D F R$, $C H S 1, C H S 2, F 3 H, F 3^{\prime} 5^{\prime} H$, and $F L S$ ). The yeast strain Y1HGold transformed with bait vector was cultured on SD -Ura medium and placed in a $30^{\circ} \mathrm{C}$ incubator for three days. Subsequently, the prey vector was transformed into the yeast strain Y1HGold previously transformed with the bait vector, and the resulting strain was plated on SD -Leu-Ura medium. The positive clones were diluted with $0.9 \% \mathrm{NaCl}$ to an OD600 of 0.1 , after which $2 \mu \mathrm{L}$ of each suspension was spotted on SD -Leu medium with or without aureobasidin A (45 $\left.\mathrm{ng} \mathrm{mL}^{-1}\right)$.

\section{Transient dual-luciferase assay}

The full-length SIBBX20 ORF was amplified by using tomato cDNA as a template and inserted into the $p$ Green II 62-SK vector. Promoter fragments from DFR (bp 1 to 1490) were cloned into the reporter vector, $p$ Green II 0800-LUC. The constructed vectors were individually introduced into Agrobacterium strain GV2260. The Agrobacterium liquid introduced into the reporter vector and effect vector were mixed and injected into tobacco leaves. Transient expression was evaluated three days after infiltration ${ }^{55}$. The firefly luciferase activity was detected by a dual-luciferase reporter assay system (Promega, USA).

\section{Electrophoretic mobility shift assays (EMSAs)}

The $S l B B X 20$ gene was cloned into $p E T 28 a$ to express the His-tagged SlBBX20 protein. Based on the DFR promoter sequence, distinct 30-bp single-stranded fragments containing the cis-acting elements AACGTG or CACATGG were synthesized (TsingKe, China) and labeled using the Biotin $3^{\prime}$ End DNA Labeling Kit (Thermo Scientific, USA). The cis-acting element was replaced with a series of guanosines to obtain the mutated fragment. The labeled DNA fragment and purified HisSIBBX20 protein were incubated in the reaction mixture for $30 \mathrm{~min}$ as described previously ${ }^{18}$. The protein-DNA complexes were separated in $6.5 \%$ native PAGE gels. The gels were transferred to a nylon membrane (Beyotime Biotechnology, China). After UV cross-linking, chemiluminescence was used to observe the migration of the biotin-labeled probe on the membranes.

\section{Yeast two-hybrid assay}

The SlBBX20 coding sequence was inserted into the prey vector $p G B K T 7$ (BD) to yield $B D-S l B B X 20$, which was used as bait to screen a tomato yeast two-hybrid library, which showed that SlCSN5-2 and SlBBX20 
interact. Furthermore, the coding sequence for a truncated SICSN5-2 protein construct lacking 56 amino acids at its N-terminus (residues 57 to 367 , SlCSN5-2 $57-367$ ) was amplified and cloned into the bait vector $p G A D T 7$ (AD) to yield $A D-S l C S N 5-2$. The $B D-S 1 B B X 20$ and $A D$ S1CSN5-2 plasmids were cotransformed into yeast strain AH109. After transformation, yeast AH109 cells were grown on SD -Trp-Leu medium for 3 days. A single clone was spotted in SD -Trp-Leu-His-Ade medium, and the growth of yeast cells was observed. The $p G B K T 7$ and $A D$-SlCSN5-2 plasmids or $B D-S l B B X 20$ and $p G A D T 7$ plasmids were used as negative controls.

\section{Bimolecular fluorescence complementation (BiFC)}

The SIBBX2O coding sequence was inserted into $p H B T$ $n Y F P$ to yield $p H B T-S I B B X 20-n Y F P$. The full-length SICSN52 coding sequence was cloned into $p H B T-c Y F P$ to yield $p H B T-S I C S N 5-2-c Y F P$. Two plasmids, $p H B T-S 1 B B X 20-$ $n Y F P$ and $p H B T-S 1 C S N 5-2-c Y F P$, were cotransformed into Arabidopsis protoplasts. After the protoplasts had been cultured for $12 \mathrm{~h}$, YFP fluorescence was observed by confocal microscopy.

\section{Coimmunoprecipitation}

For coimmunoprecipitation assays, tobacco protoplasts coexpressing SIBBX20-HA and SlCSN5-2-FLAG or expressing $\mathrm{Mer}$ as a control were collected, resuspended in extraction buffer and centrifuged at $12000 \times \mathrm{g}$ at $4{ }^{\circ} \mathrm{C}$ for $10 \mathrm{~min}$. Five microliters of anti-FLAG matrix beads (Sigma, USA) were added to the supernatant and incubated at $4{ }^{\circ} \mathrm{C}$ for $2 \mathrm{~h}$ to capture the epitope-tagged protein. Finally, antiHA (MBL, Japan) or anti-FLAG (Sigma, USA) antibodies were used for western blot analysis. Anti-UBQ (Millipore, USA) was used to detect ubiquitination of the SlBBX20 protein, and anti-actin was used as a control.

\section{Subcellular localization}

The SlCSN5-2 coding sequence without a stop codon was amplified and cloned into $p H B T$-GFP using genespecific primers (Supplementary Tables S1). Tobacco protoplasts were extracted and cotransformed with the SlCSN5-2-GFP plasmid and a nuclear marker fused to RFP. After expression in protoplasts for $10 \mathrm{~h}$, fluorescence was observed under a laser scanning confocal microscope.

\section{Downregulation of CSN5 expression by virus-induced gene silencing (VIGS)}

Because the two CSN5 sequences in tomato (CSN5-1 and CSN5-2) and two CSN5B sequences in tobacco (CSN5B-1 and CSN5B-2) are highly homologous, it is difficult to individually interfere with the two CSN5 sequences in a single species. Therefore, we elected to interfere with the expression of both. We employed virus-induced gene silencing (VIGS) and stable RNA interference (RNAi) to downregulate the expression of CSN5 in tobacco and tomato, respectively. For VIGS, we selected a unique sequence from $N b C S N 5 B$ and ligated the sequence into pTRV2. The recombinant plasmid was transferred into Agrobacterium GV3101. Agrobacterium strains transformed with $N b C S N 5 B-T R V 2$ and TRV1 or TRV2 and TRV1 were mixed and injected into tobacco leaves. After 10 days, the protoplasts were extracted from the tobacco, and SlBBX20$H A$ was transiently expressed. After SlBBX20-HA had been expressed in the protoplasts for $10 \mathrm{~h}$, SlBBX20-HA was extracted and detected by western blotting. A unique sequence was selected from SlCSN5 and used to construct an RNAi vector for stable transformation. The sequences of the gene-specific oligonucleotides used in the analysis are listed in Supplementary Table S1.

\section{Statistical analysis}

Statistical analyses were performed using Prism 6 and SPSS 26.0. All of the experiments were repeated at least three times. Statistically significant differences were determined by subjecting the data to one-way ANOVA. The data are reported as the mean value \pm SE. * indicates $P<0.05$, and ${ }^{* *}$ indicates $P<0.01$.

\section{Acknowledgements}

This work was supported by grants from the Fundamental Research Funds for the Central Universities (2662019PY048) and the National Natural Science

Foundation of China (31772313, 31972421, and 31991182). We thank Professor Robert M. Larkin and Xiangzong Meng for critical reading of our manuscript.

\section{Author contributions}

T.W., D.L., H.P. and Z.Y. conceived and designed the research. D.L., A.L., C.Z. and W.S. performed the experiments and carried out the fieldwork. D.L. and C.X. analyzed the data and wrote the manuscript. J.Z., C.Y., Y.L., H.L., H.P. and T.W. provided advice related to the research. All the authors have confirmed the final version of the manuscript.

Conflict of interest

The authors declare no competing interests.

Supplementary information The online version contains supplementary material available at https://doi.org/10.1038/s41438-021-00595-y.

Received: 27 October 2020 Revised: 24 March 2021 Accepted: 19 April 2021 Published online: 01 July 2021

\section{References}

1. Perez-Diaz, J. R. et al. New member of the R2R3-MYB transcription factors family in grapevine suppresses the anthocyanin accumulation in the flowers of transgenic tobacco. Plant Mol. Biol. 90, 63-76 (2016).

2. Fang, H. et al. The B-box zinc finger protein MdBBX20 integrates anthocyanin accumulation in response to ultraviolet radiation and low temperature. Plant Cell Environ. 42, 2090-2104 (2019).

3. An, J. P. et al. An Apple B-Box Protein MdBBX37 modulates anthocyanin biosynthesis and hypocotyl elongation synergistically with MdMYBs and MdHY5. Plant Cell Physiol. 61, 130-143 (2020).

4. An, J. P. et al. MdBBX22 regulates UV-B-induced anthocyanin biosynthesis through regulating the function of MdHY5 and is targeted by MdBT2 for 265 proteasome-mediated degradation. Plant Biotechnol. J. 17, 2231-2233 (2019). 
5. Bai, S. et al. BBX16, a B-box protein, positively regulates light-induced anthocyanin accumulation by activating MYB10 in red pear. Plant Biotechnol. J. 17, 1985-1997 (2019).

6. Bai, S. et al. Two B-box proteins, PpBBX18 and PpBBX21, antagonistically regulate anthocyanin biosynthesis via competitive association with Pyrus pyrifolia ELONGATED HYPOCOTYL 5 in the peel of pear fruit. Plant J. 100 1208-1223 (2019)

7. Peng, T. et al. Screening of UV-B-induced genes from apple peels by $\mathrm{SSH}$ : possible involvement of MdCOP1-mediated signaling cascade genes in anthocyanin accumulation. Physiol. Plant 148, 432-444 (2013).

8. Jakkola, L. New insights into the regulation of anthocyanin biosynthesis in fruits. Trends Plant Sci. 18, 477-483 (2013).

9. Yan, S. et al. Anthocyanin Fruit encodes an R2R3-MYB transcription factor, SIAN2-like, activating the transcription of SIMYBATV to fine-tune anthocyanin content in tomato fruit. N. Phytol. 225, 2048-2063 (2020).

10. Colanero, S., Tagliani, A., Perata, P. \& Gonzali, S. Alternative splicing in the anthocyanin fruit gene encoding an R2R3 MYB transcription factor affects anthocyanin biosynthesis in tomato fruits. Plant Commun 1, (2020).

11. Sun, C. et al. A transcriptional network promotes anthocyanin biosynthesis in tomato flesh. Mol. Plant 13, 42-58 (2020).

12. Zhang, Y., Butelli, E. \& Martin, C. Engineering anthocyanin biosynthesis in plants. Curr. Opin. Plant Biol. 19, 81-90 (2014).

13. Hichri, I. et al. The basic helix-loop-helix transcription factor MYC1 is involved in the regulation of the flavonoid biosynthesis pathway in grapevine. Mol. Plant 3, 509-523 (2010)

14. Kiferle, C. et al. Tomato R2R3-MYB proteins SIANT1 and SIAN2: same protein activity, different roles. PloS ONE 10, e0136365 (2015).

15. Jian, W. et al. SIMYB75, an MYB-type transcription factor, promotes anthocyanin accumulation and enhances volatile aroma production in tomato fruits. Hortic. Res. 6, 22 (2019).

16. Mathews, $\mathrm{H}$. et al. Activation tagging in tomato identifies a transcriptional regulator of anthocyanin biosynthesis, modification, and transport. Plant Cell 15, 1689-1703 (2003)

17. Espley, R. V. et al. Red colouration in apple fruit is due to the activity of the MYB transcription factor, MdMYB10. Plant J. 49, 414-427 (2007).

18. Hu, D. et al. MdMYB1 regulates anthocyanin and malate accumulation by directly facilitating their transport into vacuoles in apples. Plant Physiol. 170, 1315-1330 (2016)

19. Wang, J. et al. Anthocyanin biosynthesis regulation in the fruit of Citrus sinensis cv. Tarocco. Plant Mol. Biol. Rep. 34, 1043-1055 (2016).

20. $\mathrm{Xu}, \mathrm{H}$. et al. The molecular mechanism underlying anthocyanin metabolism in apple using the MdMYB16 and MdbHLH33 genes. Plant Mol. Biol. 94, 149-165 (2017).

21. Xie, X. B. et al. The bHLH transcription factor MdbHLH3 promotes anthocyanin accumulation and fruit colouration in response to low temperature in apples. Plant Cell Environ. 35, 1884-1897 (2012).

22. An, X., Tian, Y., Chen, K., Wang, X. \& Hao, Y. The apple WD40 protein MdTTG1 interacts with bHLH but not MYB proteins to regulate anthocyanin accumulation. Plant Physiol. 169, 710-717 (2012).

23. Khanna, R. et al. The Arabidopsis B-box zinc finger family. Plant cell 21, 3416-3420 (2009)

24. Indorf, M., Cordero, J., Neuhaus, G. \& Rodriguez-Franco, M. Salt tolerance (STO), a stress-related protein, has a major role in light signalling. Plant J. 51, 563-574 (2007).

25. Datta, S. et al. LZF1/SALT TOLERANCE HOMOLOG3, an Arabidopsis B-box protein involved in light-dependent development and gene expression, undergoes COP1-mediated ubiquitination. Plant cell 20, 2324-2338 (2008).

26. Holtan, H. E. et al. BBX32, an Arabidopsis B-Box protein, functions in light signaling by suppressing HY5-regulated gene expression and interacting with STH2/BBX21. Plant Physiol. 156, 2109-2123 (2011).

27. Fan, X. Y. et al. BZS1, a B-box protein, promotes photomorphogenesis downstream of both brassinosteroid and light signaling pathways. Mol. Plant 5, 591-600 (2012)

28. Gangappa, S. N. et al. The Arabidopsis B-BOX protein BBX25 interacts with HY5, negatively regulating BBX22 expression to suppress seedling photomorphogenesis. Plant Cell 25, 1243-1257 (2013).

29. Wei, C. Q. et al. The Arabidopsis B-box protein BZS1/BBX20 interacts with HY5 and mediates strigolactone regulation of photomorphogenesis. J. Genet Genomics 43, 555-563 (2016).
30. Zhang, X. \& Lin, R. Light signaling differentially regulates the expression of group IV of the B-box zinc finger family. Plant Signal Behav. 12, e1365213 (2017).

31. Yadav, A. et al. The B-Box-containing microprotein miP1a/BBX31 regulates photomorphogenesis and UV-B protection. Plant Physiol. 179, 1876-1892 (2019).

32. Ou, C. et al. A 14 nucleotide deletion mutation in the coding region of the PpBBX24 gene is associated with the red skin of "Zaosu Red" pear (Pyrus pyrifolia White Pear Group): a deletion in the PpBBX24 gene is associated with the red skin of pear. Hortic Res. 7, 39 (2020).

33. Fang, $\mathrm{H}$. et al. MdCOL4 interaction mediates crosstalk between UV-B and high temperature to control fruit coloration in apple. Plant Cell Physiol. 60 1055-1066 (2019).

34. Xiong, C. et al. A tomato B-box protein SIBBX20 modulates carotenoid biosynthesis by directly activating PHYTOENE SYNTHASE 1, and is targeted for 265 proteasome-mediated degradation. N. Phytol. 221, 279-294 (2019).

35. Bursch, K. et al. Identification of BBX proteins as rate-limiting cofactors of HY5. Nat. Plants 6, 921-928 (2020).

36. Lescot, M. et al. PlantCARE, a database of plant cis-acting regulatory elements and a portal to tools for in silico analysis of promoter sequences. Nucleic Acids Res. 30, 325-427 (2002).

37. Hind, S. R. et al. The COP9 signalosome controls jasmonic acid synthesis and plant responses to herbivory and pathogens. Plant J. 65, 480-491 (2011).

38. Aharoni, A., Vos, C. H. R. D., Wein, M., Sun, Z. \& O'Connell, A. P. The strawberry FaMYB1 transcription factor suppresses anthocyanin and flavonol accumulation in transgenic tobacco. Plant J. 28, 319-332 (2010).

39. Albert, N. W., Davies, K. M., Lewis, D. H., Zhang, H. \& Schwinn, K. E. A conserved network of transcriptional activators and repressors regulates anthocyanin pigmentation in eudicots. Plant Cell Environ. 26, 962-980 (2014).

40. Martin, C., Prescott, A., Mackay, S., Bartlett, J. \& Vrijlandt, E. Control of anthocyanin biosynthesis in flowers of Antirrhinum majus. Plant J. 1, 37-49 (1991).

41. Kazama, Y. et al. Characterization of a heavy-ion induced white flower mutant of allotetraploid Nicotiana tabacum. Plant Cell Rep. 32, 11-19 (2013).

42. Chamovitz, D. A. et al. The COP9 complex, a novel multisubunit nuclear regulator involved in light control of a plant developmental switch. Cell $\mathbf{8 6}$ 115-121 (1996).

43. Wei, N., Daniel, A. C. \& Deng, X. W. Arabidopsis COP9 is a component of a novel signaling complex mediating light control of development. Cell $\mathbf{7 8}$, 117-124 (1994).

44. Gusmaroli, G., Figueroa, P., Serino, G. \& Deng, X. W. Role of the MPN subunits in COP9 signalosome assembly and activity, and their regulatory interaction with Arabidopsis Cullin3-based E3 ligases. Plant cell 19, 564-581 (2007).

45. Jin, D., Li, B., Deng, X. W. \& Wei, N. Plant COP9 signalosome subunit 5, CSN5. Plant Sci. 224, 54-61 (2014).

46. Wei, N. \& Deng, X. W. The COP9 signalosome. Annu. Rev. Cell Dev. Biol. 19, 261-286 (2003).

47. Wee, S., Geyer, R. K., Toda, T. \& Wolf, D. A. CSN facilitates Cullin-RING ubiquitin ligase function by counteracting autocatalytic adapter instability. Nat. Cell Biol. 7, 387-391 (2005).

48. Wei, N., Serino, G. \& Deng, X. W. The COP9 signalosome: more than a protease Trends Biochem Sci. 33, 592-600 (2008).

49. Peng, Z., Serino, G. \& Deng, X. W. Molecular characterization of subunit 6 of the COP9 signalosome and its role in multifaceted developmental processes in Arabidopsis. Plant cell 13, 2393-2407 (2001).

50. Chen, $H$. et al. Arabidopsis CULLIN4 forms an E3 ubiquitin ligase with RBX1 and the CDD complex in mediating light control of development. Plant cell 18, 1991-2004 (2006)

51. Biedermann, S. \& Hellmann, H. WD40 and CUL4-based E3 ligases: lubricating all aspects of life. Trends Plant Sci. 16, 38-46 (2011).

52. Rio, D. C., Ares, M., Hannon, G. J. \& Nilsen, T. W. Purification of RNA using TRlzol (TRI Reagent). Cold Spring Harb. Protoc. 2010, pdb.prot5439-pd (2010).

53. Li, W. et al. Development and systematic validation of GPCR assays for rapid and reliable differentiation of Xylella fastidiosa strains causing citrus variegated chlorosis. J. Microbiol. Methods 92, 79-89 (2013).

54. Mortazavi, A., Williams, B. A., McCue, K., Schaeffer, L. \& Wold, B. Mapping and quantifying mammalian transcriptomes by RNA-Seq. Nat. Methods 5, 621-628 (2008).

55. Hellens, R. P. et al. Transient expression vectors for functional genomics, quantification of promoter activity and RNA silencing in plants. Plant Methods 1, 13 (2005). 